\title{
- Répartition des ressources et des utilisations d'eau dans le monde : disparités présentes et futures
}

\author{
- World distribution of water ressources and utilizations : \\ present and future disparities - \\ par Jean Margat \\ Conseiller du BRGMI
}

\section{La Terre est la planète de l'eau mais la répartition entre les diffé- rents pays ou régions du globe est loin d'être équitable. Les situations de pénurie dans certaines régions risquent de s'aggraver.}

\section{INTRODUCTION}

La Terre est la planète de l'eau. Vue de Sirius, sans doute. Les donnes en eau de la nature n'en sont pas moins très inégalement réparties dans le monde, tout comme les besoins en eau humains, en partie sous l'effet d'un même facteur : les différences de climat. Cette double variété est bien connue et les médias popularisent les images de situations extrêmes, conjoncturelles ou chroniques : inondations et sécheresses en alternance, surabondance ici, pénurie là. Aussi la comparaison des ressources et des besoins, qui requiert un minimum de quantification, n'a-t-elle guère de sens à l'échelle planétaire et en situation moyenne ; elle n'est pertinente que dans des cadres plus régionaux et temporellement définis.
Cette comparaison est encore trop grossière dans la plupart des pays pris globalement, surtout les plus étendus et diversifiés. Néanmoins, malgré les inégales validités des chiffrages, les "statistiques" disponibles par pays sur les ressources et les utilisations d'eau révèlent de tels contrastes qu'elles autorisent à calculer des indicateurs macroscopiques significatifs et à esquisser une géographie d'ordres de grandeur.

L'eau n'occupe qu'une place faible, sinon nulle, dans les statistiques macroéconomiques et dans la géographie économique des matières premières et des ressources naturelles. Le bref panorama présenté vise à combler cette lacune.

\section{LES RESSOURCES EN EAU SONT TRÈS INÉGALEMENT RÉPARTIES}

Deux variables régionales continues caractérisent la genèse locale des flux d'eau naturels, seul facteur physique de la ressource que l'on soit en mesure d'estimer assez universellement et de manière assez homogène pour permettre sa description générale et rendre des comparaisons valables :

- les précipitations, qui forment déjà une ressource

1 Bureau de Recherches Géologiques et Minières.

Water resources are very irregularly distributed through the world, and so is their utilization, both between countries and between users within countries. Tables and maps describing this variety can be drawn up using available statistics and the macroscopic indicators derived from them. Water resources depend upon the climates that generate them and the physiographic structures that distribute them. Their utilization also varies according to climate, but depends in addition upon the degree of development of the country concerned. Resources vary, per head of population, in the ratio 1:1000, and utilization in the ratio 1:100.

These inequalities are likely to be aggravated in the future. The rapid decrease in physical resources per head of population caused by demographic growth risk in some instances limiting the possibility of satisfying the increasing requirements caused by the pressures of development, or at least strongly accentuating the artificialization of the terrestrial stages of the water cycle in certain regions. 
Tableau 1. - Flux d'eau terrestres répartis par zone climatique (moyennes en $\mathbf{~ k m}^{3} /$ an $=$ milliards de $\mathbf{m}^{3} / a n$ ).

\begin{tabular}{|c|c|c|c|c|c|}
\hline \multirow[t]{2}{*}{ Zones climatiques } & \multirow[t]{2}{*}{ Précipitations } & \multirow[t]{2}{*}{ Evapo-transpiration } & \multicolumn{2}{|c|}{ Ecoulement total } & \multirow{2}{*}{$\begin{array}{l}\text { Ecoulement régulier } \\
\text { (basses eaux et } \\
\text { eaux souterraines) }\end{array}$} \\
\hline & & & & $\%$ & \\
\hline Zone tempérée ( $\mathrm{N}$ et $\mathrm{S}$ ) & 49000 & 27800 & 21200 & (48) & 6500 \\
\hline Zone aride et semi-aride & 7000 & 6200 & 800 & (2) & $200^{(1)}$ \\
\hline Zone intertropicale humide & 60000 & 38000 & 22000 & $(50)$ & 6300 \\
\hline Total (Monde) & 116000 & 72000 & 44000 & $(100)$ & 13000 \\
\hline
\end{tabular}

(1) Eaux souterraines pour l'essentiel.

pour la végétation utile et les cultures "pluviales", tout en engendrant les écoulements, varient dans le monde en hauteur moyenne annuelle de moins de $1 \mathrm{~cm}$ à $10 \mathrm{~m}$, soit de moins de $10000 \mathrm{~m}^{3}$ à 10 millions de $\mathrm{m}^{3}$ par $\mathrm{km}^{2}$;

- les flux moyens locaux engendrés et écoulés (superficiels ou souterrains) par unité de surface varient entre quelques milliers de $\mathrm{m}^{3} / \mathrm{an}$ par $\mathrm{km}^{2}$ - en zone très aride à plus de trois millions de $\mathrm{m}^{3} / \mathrm{an}$ par $\mathrm{km}^{2}$ (jusqu'à quatre millions localement) dans les régions les plus arrosées, soit un rapport de plus de 1 à 1000 entre les extrêmes. En outre, ces écoulements sont très changeants suivant les années, et d'autant plus qu'ils sont faibles en moyenne.

Sur les quelques $44000 \mathrm{~km}^{3}$ annuels écoulés, bon an mal an, sur l'ensemble des terres émergées, la première inégalité et la plus flagrante est leur répartition entre les grandes zones climatiques. Les zones arides et semi-arides du monde entier ne reçoivent que $6 \%$ des précipitations globales et il ne s'y forme que $2 \%$ de l'écoulement terrestre ; les zones humides tempérées et intertropicales se partagent à peu près également le reste, c'est-à-dire la plus grande part (tableau 1).

Globalisés par pays entiers, en considérant d'abord les flux (toujours en moyenne) formés sur leur territoire, les ressources en eau naturelles intérieures dépendent évidemment de l'étendue de chaque pays et des zones climatiques où il se situe, les zones tropicales humides et arctiques (ou antarctiques) étant les plus généreuses.

La gamme de ces ressources en eau naturelles "nationales" est très large et l'écart des extrêmes est énorme, de moins de 100 millions à plus de 5000 milliards de $\mathrm{m}^{3} / \mathrm{an}$. L'essai d'anamorphose cartographique, où l'eau remplace l'espace pour chaque pays (fig. 1), donne une nouvelle vision du monde, non dépourvue de signification géopolitique. Neuf pays, "géants" mondiaux des ressources en eau intérieures, sont plus de mille fois milliardaires en $\mathrm{m}^{3}$ annuels et se partagent $60 \%$ des ressources en eau naturelles mondiales.

Les neuf grands des ressources en eau (d'après I. Shiklomanov, UNESCO, 1996, ou des sources nationales) sont décrits dans le tableau 2.
Tableau 2. - Les pays les plus riches en eau.

\begin{tabular}{lll}
\hline & (en année moyenne) \\
Brésil & $:$ & $6220 \mathrm{~km}^{3} / \mathrm{an}$ \\
Russie & $:$ & $4059 \mathrm{~km}^{3} / \mathrm{an}$ \\
USA (avec Alaska) & $:$ & $3760 \mathrm{~km}^{3} / \mathrm{an}$ \\
Canada & $:$ & $3290 \mathrm{~km}^{3} / \mathrm{an}$ \\
Chine & $:$ & $2800 \mathrm{~km}^{3} / \mathrm{an}$ \\
Indonésie & $:$ & $2530 \mathrm{~km}^{3} / \mathrm{an}$ \\
Inde & $:$ & $1850 \mathrm{~km}^{3} / \mathrm{an}$ \\
Colombie & $:$ & $1200 \mathrm{~km}^{3} / \mathrm{an}$ \\
Pérou & $:$ & $1100 \mathrm{~km}^{3} / \mathrm{an}$
\end{tabular}

En comparaison, ensemble des 15 pays

de l'Union Européenne : $\quad 1171 \mathrm{~km}^{3} / \mathrm{an}$.

Tableau 3. - Pays les plus dépourvus de ressources en eau.

\begin{tabular}{|lcl|}
\hline Koweit, Bahrein & $:$ & $\begin{array}{l}\text { ressources renouvelables } \\
\text { quasi nulles en eau douce }\end{array}$ \\
Malte & $:$ & 15 millions de $\mathrm{m}^{3} / \mathrm{an}^{(2)}$ \\
Gaza & $:$ & 46 millions de $\mathrm{m}^{3} / \mathrm{an}$ \\
Emirats Arabes Unis & $:$ & 500 millions de $\mathrm{m}^{3} / \mathrm{an}$ \\
Libye & $:$ & 600 millions de $\mathrm{m}^{3} / \mathrm{an}$ \\
Singapour & $:$ & 600 millions de $\mathrm{m}^{3} / \mathrm{an}$ \\
Jordanie & $:$ & 680 millions de $\mathrm{m}^{3} / \mathrm{an}$ \\
Israël & $:$ & 750 millions de $\mathrm{m}^{3} / \mathrm{an}$ \\
Chypre & $:$ & 900 millions de $\mathrm{m}^{3} / \mathrm{an}$ \\
\hline
\end{tabular}

(2) Eau douce exploitable au maximum sans risque de rupture d'équilibre avec l'eau de mer.

A l'autre extrême, les pays les plus dépourvus, dont les ressources ne se chiffrent qu'en millions de $\mathrm{m}^{3} / \mathrm{an}$, sont les plus petits (notamment insulaires) et/ou les plus arides (tableau 3 ). 


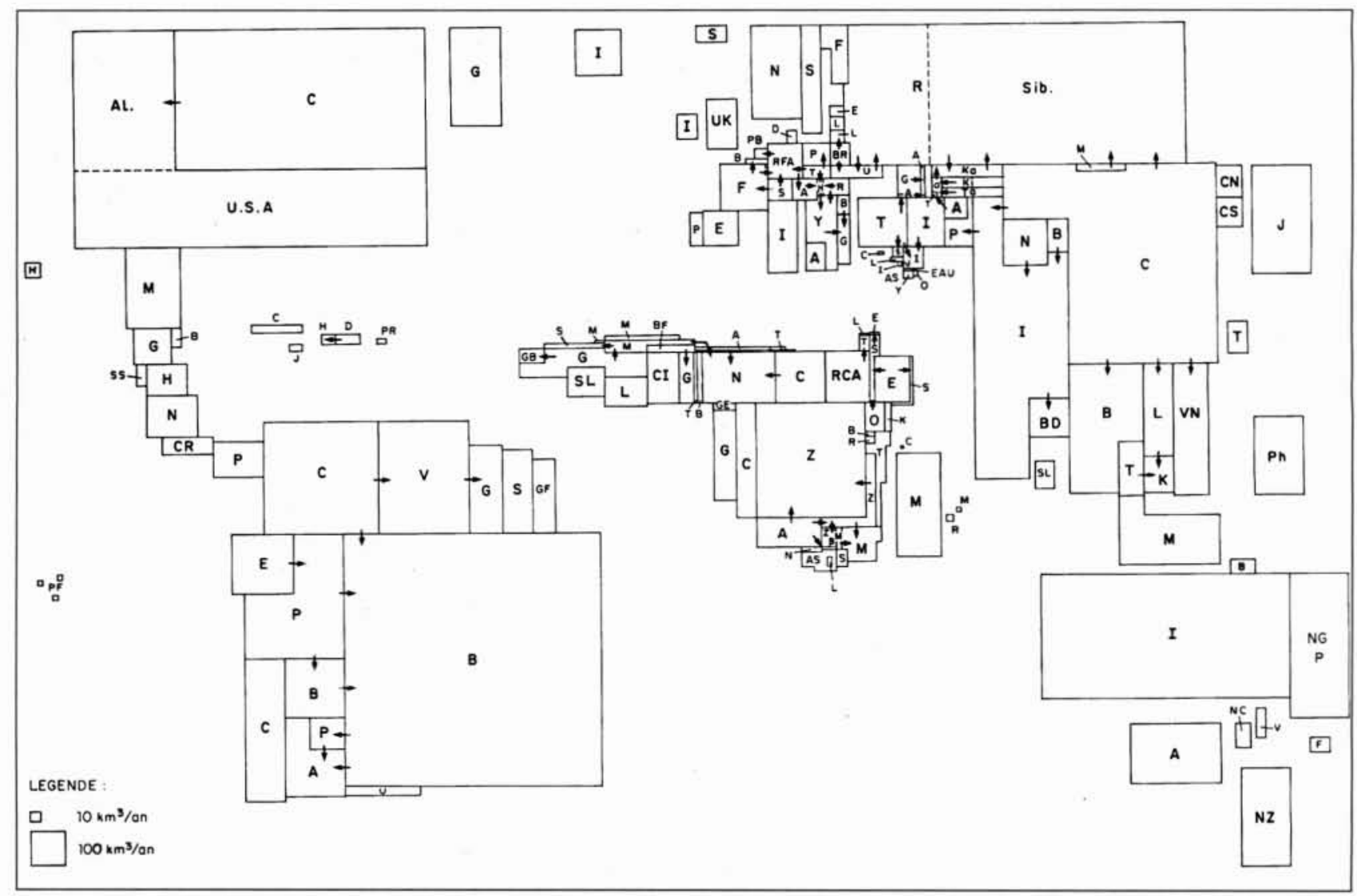

Fig. 1 - Ressources en eau naturelles intérieures de chaque pays (Flux moyens annuels). Anamorphose cartographique (d'après les données disponibles en 1990).

La plupart des pays industrialisés européens de taille moyenne disposent chacun de 100 à 200 milliards de $\mathrm{m}^{3} / \mathrm{an}$ de ressource propre (France : $170 \mathrm{~km}^{3} / \mathrm{an}$ ) et le Japon de $435 \mathrm{~km}^{3} / \mathrm{an}$.

Beaucoup de pays reçoivent en outre des eaux apportées spontanément par des fleuves trans-frontaliers, suppléments notables, parfois supérieurs à leur ressource intérieure et potentialités à prendre en compte, malgré les contraintes géopolitiques qui peuvent s'y attacher. Le Brésil passe ainsi à 8120 milliards de $\mathrm{m}^{3} / \mathrm{an}$, la Russie à 4286 , le Bangladesh de 357 à 1357, l'Argentine de 270 à 893.

En agrégeant les données nationales en une douzaine de grands espaces géopolitiques, ce qui améliore la validité des arrondis et gomme une grande partie des échanges d'eau transfrontaliers (tabl. 5), des écarts significatifs apparaissent encore.

Pour corriger l'incidence des grandes différences de densité de population des pays, les ressources (intérieures et extérieures) sont à rapporter aux populations et à exprimer en flux moyen par habitant, ou encore, à l'inverse en "densité de population rapportée aux ressources"(2), exprimée en nombre d'habitants par million de $\mathrm{m}^{3} / \mathrm{an}$, ce qui n'annule pas cependant les effets d'échelle. Mieux que les statistiques globales précédentes, ces ratios caractérisent sans doute plus valablement la richesse ou la pauvreté en eau des pays. Les ressources en eau sont encore plus inégalement partagées entre les hommes qu'entre les pays, car il y a très peu de lien entre les densités de population et les ressources en eau de chaque territoire. Rapportées aux populations de 1995, les plus fortes ressources en eau par habitant s'observent dans des pays équatoriaux ou nordiques à eaux très abondantes et/ou peu peuplés : le record du monde paraît être la Guyane française (plus d'un million de $\mathrm{m}^{3} / \mathrm{an}$ ) et l'Islande est le record d'Europe $\left(630000 \mathrm{~m}^{3} / \mathrm{an}\right)$. Dans près de dix pays la ressource par habitant dépasse $100000 \mathrm{~m}^{3} / \mathrm{an}$. La faible densité de population pouvant l'emporter sur la modicité du flux, des ressources per capita sont parfois élevées dans certains pays ou territoires arides : $760000 \mathrm{~m}^{3} / \mathrm{an}$ dans le Territoire du Nord d'Australie, $29000 \mathrm{~m}^{3} / \mathrm{an}$ dans le Haut-Colorado (en 1990) aux EtatsUnis, par exemple.

A l'autre extrême, les plus faibles ressources naturelles par habitant se trouvent en pays de la zone aride ou insulaires plus ou moins peuplés, à ressource globale très limitée. Les dix plus pauvres, parmi lesquels se trouvent sept pays arabes, sont, en se référant aux populations de 1995, décrits dans le tableau 4.

(2) Ou "index de compétition" de M. Falkenmark (1986). 
Tableau 4. - Pays qui offrent le moins de ressources en eau par habitant.

\begin{tabular}{|lc|}
\hline & $\mathrm{m}^{3} / \mathrm{an}$ par habitant \\
Koweit & $\varepsilon$ \\
Malte & $40^{*}$ \\
Qatar & 54 \\
Gaza & 59 \\
Bahamas & 75 \\
Arabie Saoudite & 105 \\
Libye & 111 \\
Bahraïn & 185 \\
Jordanie & 185 \\
Singapour & 211 \\
Emirats Arabes Unis & 279 \\
\hline
\end{tabular}

* eau douce exploitable sans risque de rupture d'équilibre avec eau salée, seule.
Entre ces pays à situations extrêmes se placent la plupart des pays développés de la zone tempérée aux ressources de l'ordre de quelques milliers de $\mathrm{m}^{3} / \mathrm{an}$ par habitant (France : 3133 ; ensemble des quinze pays de l'Union européenne : 3467 en 1995).

D'une gamme de situations réelles aussi étendue pourrait se déduire une échelle conventionnelle des richesses en eau relatives, dont les deux classes centrales correspondraient au groupe de pays le plus nombreux :

\begin{tabular}{|lc|}
\hline & $\mathrm{m}^{3} / \mathrm{an}$ par habitant \\
pléthore & 100000, voire 1000000 \\
abondance & 10000 à 100000 \\
suffisance & 2000 à 10000 \\
modicité & 1000 à 2000 \\
pauvreté & 500 à 1000 \\
dénuement & $<500$ \\
&
\end{tabular}

Tableau 5. - Ressources en eau douce naturelles et renouvelables dans le monde (3).

\begin{tabular}{|c|c|c|c|c|}
\hline $\begin{array}{l}\text { Espaces géopolitiques } \\
\text { (groupes de pays) }\end{array}$ & $\begin{array}{c}\text { Ressources } \\
\text { intérieures } \\
\text { moyenne en } \\
\mathrm{km}^{3} / \mathrm{an}^{*}\end{array}$ & $\begin{array}{c}\text { Ressources } \\
\text { d'origine } \\
\text { extérieure au } \\
\text { groupe de pays } \\
\text { en } \mathrm{km}^{3} / \mathrm{an}\end{array}$ & $\begin{array}{c}\text { Part } \\
\text { relativement } \\
\text { régulière } \\
\text { (superficielle } \\
\text { et souterraine) } \\
\text { en } \mathbf{~ k m}^{3} / \mathrm{an}\end{array}$ & $\begin{array}{c}\text { Proportion } \\
\text { disponible } \\
\text { actuelle } \\
\text { compte tenu } \\
\text { des prélèvements } \\
\text { bruts } \\
\text { en } \%\end{array}$ \\
\hline $\begin{array}{l}\text { Europe de l'Ouest, nordique et } \\
\text { méditerranéenne (Union euro- } \\
\text { péenne+A.E.L.E. et Chypre) }\end{array}$ & 1750 & 20 & 600 & 85 \\
\hline $\begin{array}{l}\text { Europe de l'Est (avec Russie } \\
\text { d'Asie) }\end{array}$ & 4720 & 290 & 1200 & 94,5 \\
\hline $\begin{array}{l}\text { Amérique du Nord } \\
\text { (USA et Canada) }\end{array}$ & 6750 & 0 & 1800 & 92,5 \\
\hline $\begin{array}{l}\text { Amérique centrale } \\
\text { (avec Caraỉbes) }\end{array}$ & 1200 & 3 & 700 & 90 \\
\hline Amérique du Sud & 11740 & 0 & 3500 & 98,7 \\
\hline $\begin{array}{l}\text { Monde arabe, Afrique du } \\
\text { Nord (avec Soudan), Proche } \\
\text { et Moyen Orient (avec Israël) }\end{array}$ & 140 & 190 & 100 & 30 \\
\hline $\begin{array}{l}\text { Afrique au Sud du Sahara } \\
\text { (avec Madagascar) }\end{array}$ & 3910 & 0 & 1400 & 98,5 \\
\hline $\begin{array}{l}\text { Asie centrale et occidentale } \\
\text { (avec Turquie, Transcaucasie } \\
\text { et Iran) }\end{array}$ & 660 & 27 & 200 & 53 \\
\hline $\begin{array}{l}\text { Sous-continent indien et Asie } \\
\text { du Sud-Est }\end{array}$ & 7700 & 1000 & 1800 & 87,5 \\
\hline $\begin{array}{l}\text { Chine (avec Mongolie et } \\
\text { Corée du Nord) }\end{array}$ & 2900 & 0 & 1000 & 82 \\
\hline Japon et "dragons" & 590 & 0 & 200 & 76 \\
\hline Australasie et Océanie & 1690 & 0 & 500 & 98 \\
\hline Monde entier & 43750 & - & 13000 & 92 \\
\hline
\end{tabular}

* dont une partie peut être commune à plusieurs pays du groupe et une partie peut être apportée à un groupe voisin.

(3) Chiffrages actualisés en priorité d’après les évaluations nationales les plus récentes. complétées par des compilations internationales (notamment Shiklomano, UNESCO, 1996 et FAO 1995. 1997). Ils sont un peu supérieurs à ceux publiés antérieurement. 
Aux limites 1000 et $500 \mathrm{~m}^{3} / \mathrm{an}$ correspondent respectivement les seuils de pauvreté ("water stress") et de pénurie ("water scarcity") proposés par M. Falkenmark (1986) ; $1000 \mathrm{~m}^{3} / \mathrm{an}$ de ressource naturelle par habitant, en année moyenne, peuvent être considérés comme un minimum vital dans les pays à climat rendant l'irrigation nécessaire.

Sur cette base se dessine une géographie très contrastée des ressources en eau naturelles relativisées aux populations, dont la carte de la figure 2 donne une image schématique.

Cette approche quantitative des ressources en eau, identifiées aux seuls flux moyens annuels, dans une optique purement hydrologique, est pourtant non seulement trop globale - pour la plupart des pays - mais surtout trop indifférenciée. Les disparités s'accentuent d'abord à l'intérieur de beaucoup de pays à climats et à densités de population contrastés, suivant les régions ; elles s'aggravent encore si l'on considère d'autres caractères des ressources plus différenciés : conditions d'accès et de maîtrise, suivant les structures physiographiques et les régimes - dont dépendent largement les coûts de mobilisation -, qualités des eaux, degré d'autonomie.

Les flux d'eau naturels sont très diversement "organisés", concentrés et distribués par les structures hydrographiques et hydrogéologiques :

- Suivant leur taille, les bassins fluviaux collectent et groupent des parts très inégales de l'écoulement formé sur la surface terrestre. Le bassin d'un seul fleuve géant, l'Amazone, occupe près du vingtième des terres émergées et concentre $15 \%$ de l'écoulement total du monde, tandis que les dix fleuves suivants, par ordre d'abondance, drainent près de $14 \%$ des continents et $18 \%$ de tout l'écoule- ment mondial et que des milliers de fleuves et rivières se partagent le reste. Les plus grands bassins s'étendant généralement sur plusieurs pays, les ressources qu'ils offrent s'évaluent dans des cadres plurinationaux et posent des problèmes de partage : ces bassins couvrent ensemble la moitié des continents (fig. 3 ) et $60 \%$ de tout l'écoulement mondial s'y forme, en constituant des ressources en eau communes. Les autres bassins sont au contraire les unités élémentaires de l'évaluation des ressources nationales. Cet effet d'échelle vaut surtout en zones humides, car il est atténué en zone aride par la dégradation de fonctionnement qui morcelle les grands bassins fluviaux.

- Suivant la nature des roches et la structure de l'écorce terrestre, le sous-sol est doté très inégalement de réservoirs aquifères capables de régulariser les écoulements de surface et offrant en même temps des ressources permanentes, diversement abondantes et accessibles. Ces aquifères sont, eux aussi, de tailles très variées ; les plus étendus peuvent dépasser un million de $\mathrm{km}^{2}$ et se partager entre plusieurs pays, tandis que la plupart des autres sont enchassés chacun dans un même bassin fluvial.

A l'inégalité spatiale s'ajoute celle des conjonctures du temps. L'expression de flux moyens annuels, qui intègrent et nivellent des variabilités très diverses, appauvrit l'évaluation en masquant de grandes différences d'apports réels, tant au long de l'année que d'une année à l'autre. Si l'on définit cette variabilité par le rapport de l'écart moyen à la "normale" exprimé en pourcentage, on observe, par exemple, que celle des précipitations annuelles, inférieure à $20 \%$ en zone tempérée ou tropicale humide, excède 20 à $30 \%$ en zone semiaride et atteint 40 à $100 \%$ en zone aride où les "moyennes annuelles" n'ont guère de sens. De même les écoulements annuels, assez réguliers en zones humides peuvent varier de

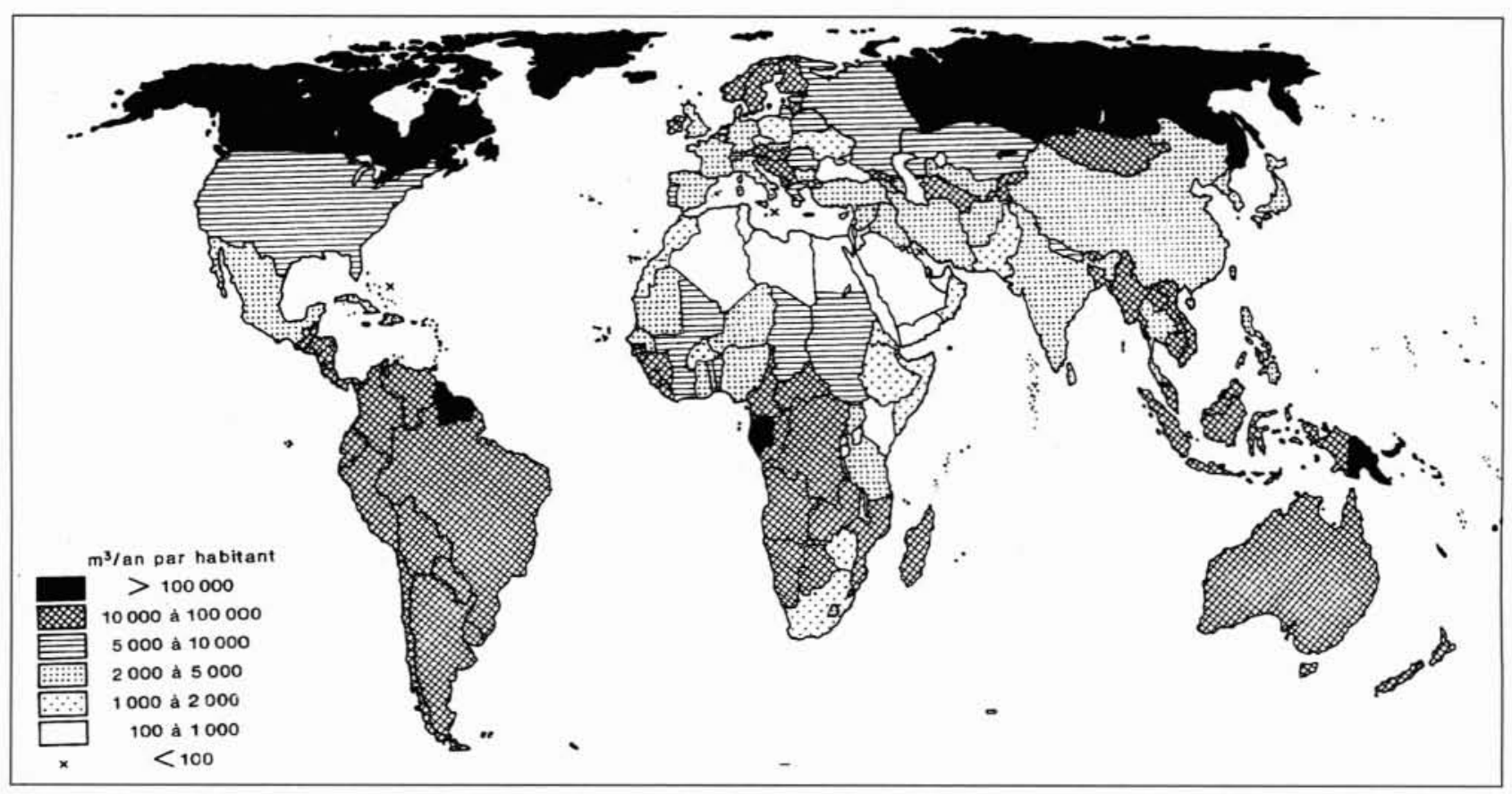

Fig. 2 - Pays classés suivant leurs ressources en eau naturelles renouvelables, internes et externes, moyennes annuelles, par habitant (populations 1995). 


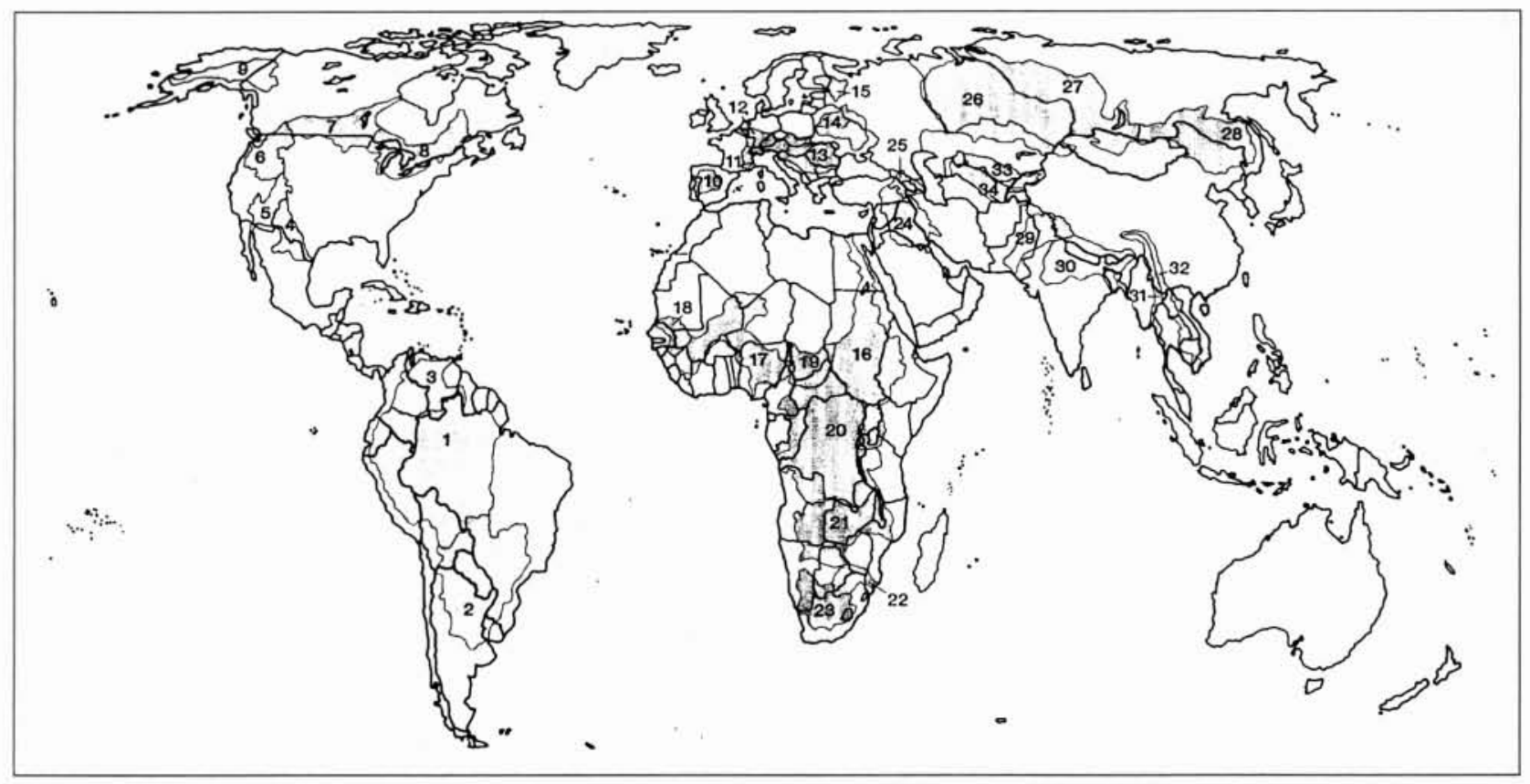

Fig. 3 - Principaux bassins fluviaux pluri-nationaux dans le monde

(1) Amazone - (2) La Plata - (3) Orénoque - (4) Rio Grande - (5) Colorado - (6) Columbia - (7) Nelson - (8) Saint-Laurent - (9) Yukon - (10) Douro, Tage et Guadiana - (11) Rhône - (12) Rhin - (13) Danube - (14) Dniepr - (15) Neva - (16) Nil - (17) Niger - (18) Sénégal - (19) Chari - (20) Zaïre - (21) Zambèze - (22) Limpopo - (23) Orange - (24) Shatt el Arab (Tigre, Euphrate) - (25) Araxe - (26) Ob (27) lénisséi - (28) Amour - (29) Indus - (30) Gange et Brhamapoutre - (31) Salouen - (32) Mékong - (33) Syr Daria - (34) Amou Daria.

1 à 2 ou 1 à 5 , voire 1 à 10 en régions à climat méditerranéen ou semi-aride, avec l'aggravation de séquences pluriannuelles de sécheresse plusieurs fois par siècle. Les distributions saisonnières varient en même temps que l'irrégularité pluriannuelle.

Les ressources en eau ne sont pas offertes seulement par des flux plus ou moins irréguliers mais aussi par des stocks qui concourent à régulariser les écoulements : les réservoirs naturels, lacs, aquifères souterrains, masses glaciaires, sont eux aussi très inégalement distribués dans le monde. Un seul lac, le Baîkal, recèle $25 \%$ du stock d'eau douce superficielle terrestre... Les réserves locales d'eau souterraine varient entre 0,1 et 10 millions de $\mathrm{m}^{3}$ par $\mathrm{km}^{2}$ et la gamme des réservoirs aquiferes va des millions de $\mathrm{m}^{3}$ aux milliers de milliards de $\mathrm{m}^{3}$. De plus, les réservoirs les plus volumineux, à stocks d'eau fossile considérables, constituent des "gisements d'eau" dont des dizaines ou des centaines de milliards de $\mathrm{m}^{3}$ sont exploitables et qui offrent des ressources en eau non renouvelables particulièrement appréciables dans certains pays arides (Sahara, Arabie, Australie...).

Toutes ces disparités se répercutent sur les ressources en eau que l'on cherche à quantifier dans des cadres régionaux ou nationaux. Elles sont encore amplifiées par les différences de travail, donc de coûts, que la maîtrise et la mobilisation de l'eau nécessitent, effort très varié selon l'accessibilité et la régularité d'occurrence de l'eau dans le milieu. Autrement dit, les potentialités en eau de la nature déjà inégalement réparties sont aussi inégalement exploitables, ou du moins sont exploitables à des coûts très inégaux, en sachant que cette exploitabilité n'est pas un caractère univoque ni absolu, mais relatif aux critères économiques des exploitants, donc à leurs objectifs d'utilisation : elle est révisable et évolutive.

Les écarts de qualités des eaux s'ajoutent aux différences de quantités et amplifient les disparités des ressources, en pouvant réduire beaucoup en certains pays les quantités d'eau utilisable. Dans un cas extrême, comme celui du Koweit, les ressources en eau douce sont quasi nulles.

Toutes ces différences accentuent encore les inégalités : les ressources les plus rares en moyenne sont souvent aussi les plus irrégulières et les plus défectueuses en qualité. Ces faiblesses de ressources se cumulent particulièrement dans les pays de la zone aride et semi-aride.

Enfin, les ressources en eau des pays sont très inégalement indépendantes : la proportion des ressources naturelles d'origine externe, faible ou nulle en beaucoup de pays (France : $8 \%$ ), peut être notable ou majeure dans certains pays peu étendus ou situés en zone aride et récepteurs de grands fleuves transfrontières (Iraq : $65 \%$; Pays-Bas : $89 \%$; Egypte : $99 \%$ ). 
Les ressources en eau des différents pays n'ont donc pas la même sécurité à long terme, car les parts externes sont sensibles aux utilisations dans les pays amont. Les différences d'autonomie des ressources ont donc une dimension géopolitique. Réciproquement les rôles de fournisseur de certains pays peuvent aussi limiter leur liberté d'action sur leurs ressources propres.

Les eaux de la nature sont encore inégalement mobilisables suivant des critères environnementaux, en fonction de l'acceptabilité des impacts de leur prélèvement - et des retours après usages, qui sont rarement sans nuisance -.

En somme, que ce soit pour des raisons pratiques et socio-économiques, géopolitiques ou écologiques, toutes les eaux de la nature, renouvelées par le cycle de l'eau, ne peuvent plus être entièrement identifiées à des ressources dévolues à l'humanité.

\section{E LES UTILISATIONS D'EAU SONT DIVERSIFIÉES ET AUSSI INÉGALES DANS LE MONDE}

La variété des utilisations de l'eau est trop connue pour qu'on la détaille ici : de la vie domestique à la plupart des secteurs économiques, il est peu d'activité humaine consommatrice ou productrice qui ne soit utilisatrice d'eau, sans substitut pour la plupart de ses usages.

Faut-il pour autant parler de besoins en eau ? Le concept théorique et normatif de besoin sert plutôt de référence pour apprécier si les demandes réelles actuelles sont insuffisantes (restrictions) ou excessives (gaspillage), ou encore pour projeter des demandes futures. On sait combien les besoins diffèrent suivant les usages, en volume comme en qualité voulue, aussi bien qu'en proportion consommée nette.

Même pour chaque usage pris à part, une grande variété de demandes unitaires - par usager, par unité de produit, par hectare irrigué... - se rencontre dans le monde et dans chaque pays, suivant les comportements des usagers, les conditions climatiques, les procédés industriels ou les pratiques d'arrosage. Aussi des standards moyens en cette matière traduisent-ils mal des réalités diversifiées.

A l'échelle macroscopique des pays elle-même, la diversité et la multiplicité des facteurs et des motifs de demandes en eau - ou plus largement de sollicitations des eaux du milieu naturel - les rendent presque aussi variées dans le monde que les ressources, qu'on globalise ces demandes par pays ou qu'on les rapporte aux populations ou à des indicateurs macro-économiques.

Trois facteurs principaux déterminent cette variété : - D'abord les climats, qui nécessitent ou non l'irrigation des cultures suivant les régions. Dans une grande partie du monde à "déficit hydrique" - zones à climat aride, semi-aride ou méditerranéen - la production agricole est subordonnée à l'irrigation, en principal ou en complément. La demande en eau d'irrigation l'emporte, souvent de beaucoup, sur toutes les autres et amplifie considérablement la demande en eau globale. L'aridité du climat grossit aussi beaucoup la consommation d'eau des rete- nues par évaporation. Ainsi, par rapport aux pays à climat humide, les pays des zones arides ou semi-arides sont doublement pénalisés : par la rareté des ressources et par l'amplification des besoins humains.

- Puis les grandeurs des populations, même si les demandes par habitant effectives varient largement, notamment en fonction des taux d'urbanisation et des niveaux de vie.

- Enfin, les formes et les degrés de développement socio-économique, qui influencent fortement les demandes des collectivités et des secteurs de production industrielle ou énergétique, ainsi que la création des moyens de les satisfaire. Un haut niveau de développement induit des demandes en eau effectives - et non pas seulement des besoins théoriques - plus fortes, sauf en agriculture (là où l'irrigation est nécessaire ou avantageuse) dans la mesure où les modes d'irrigation plus efficients (mais plus coûteux) peuvent atténuer les demandes. Toutefois, les interférences des autres facteurs brouillent la relation entre les indicateurs du développement et les demandes en eau globales, comme on le verra.

La connaissance des demandes en eau actuelles est encore bien inégale suivant les pays et les secteurs d'utilisation, car elles sont plus souvent estimées par les voies indirectes que recensées. Les statistiques disponibles ne portent généralement que sur ce qu'on sait le moins mal compter : les volumes prélevés ou produits. Encore sontelles frappées d'approximations variées qui rendent les sommations et comparaisons difficiles. Du fait des multiples "dimensions" des demandes en eau, globaliser ces seuls volumes confondus simplifie à outrance l'analyse.

En outre, quelques confusions conceptuelles et terminologiques embrouillent un peu les comparaisons et les agrégations statistiques : utilisations, demandes, productions, prélèvements d'eau ne sont pas partout équivalents (encadré 1).

Encadré 1 : Utilisations, demandes, productions, prélèvements

Strictement parlant, les demandes en eau réelles (satisfaites) correspondent aux quantités d'eau utilisées (ex-situ), par l'ensemble des usagers, pouvant se répartir en classes de qualité ; elles sont l'objet des statistiques d'utilisations, indépendamment des efficiences des usages.

La couverture de ces demandes, qui englobe les pertes de stockage et de transport, équivaut aux productions d'eau brutes et correspondent aux prélèvements sur les ressources naturelles, sauf dans les pays où des productions d'eau non conventionnelles $s^{\prime} y$ ajoutent. Utilisations et demandes sont alors supérieures aux prélèvements.

Comme sujets de chiffrage dans cet article (tableaux et cartes), les demandes en eau, prises au sens large, équivalent aux quantités d'eau utilisées et produites, soit par prélèvement (pour l'essentiel), soit par des filières non conventionnelles en quelques cas. 
Tableau 6. - Utilisations actuelles d'eau dans le monde (1990 ou année proche).

\begin{tabular}{|c|c|c|c|c|c|}
\hline \multirow[t]{3}{*}{$\begin{array}{l}\text { Espaces géopolitiques } \\
\text { (groupes de pays) }\end{array}$} & \multicolumn{4}{|c|}{$\begin{array}{c}\text { Quantités d'eau utilisées* } \\
\text { (productions) }\end{array}$} & \multirow{3}{*}{$\begin{array}{l}\text { Consommation } \\
\text { finale totale estimée } * * \\
\text { en } \mathrm{km}^{3} / \mathrm{an}\end{array}$} \\
\hline & \multirow{2}{*}{$\begin{array}{c}\text { Total } \\
\text { en } \mathrm{km}^{3} / \mathrm{an}\end{array}$} & \multicolumn{3}{|c|}{ Répartition par secteur d'utilisation en \% } & \\
\hline & & $\begin{array}{l}\text { Collectivités } \\
\text { (eau potable) }\end{array}$ & $\begin{array}{l}\text { Agriculture } \\
\text { (irrigation) }\end{array}$ & $\begin{array}{l}\text { Industries } \\
\text { et énergie }\end{array}$ & \\
\hline $\begin{array}{l}\text { Europe de l'Ouest nordique } \\
\text { et méditerranéenne } \\
\text { Union européenne + A.E.L.E. } \\
\text { et Chypre }\end{array}$ & 260 & 17 & 36 & 47 & 80 \\
\hline $\begin{array}{l}\text { Europe de l'Est } \\
\text { (avec Russie d'Asie) }\end{array}$ & 255 & 17 & 22 & 61 & 100 \\
\hline $\begin{array}{l}\text { Amérique du Nord } \\
\text { (USA et Canada) }\end{array}$ & 510 & 12 & 39 & 49 & 150 \\
\hline $\begin{array}{l}\text { Amérique centrale } \\
\text { (avec Caraïbes) }\end{array}$ & 105 & 8 & 85 & 7 & 60 \\
\hline Amérique du Sud & 155 & 23 & 70 & 7 & 90 \\
\hline $\begin{array}{l}\text { Monde arabe, Afrique du } \\
\text { Nord (avec Soudan), Proche } \\
\text { et Moyen Orient (avec Israël) }\end{array}$ & $172 * * *$ & 7 & 89 & 4 & 150 \\
\hline $\begin{array}{l}\text { Afrique au Sud du Sahara } \\
\text { (avec Madagascar) }\end{array}$ & 60 & 18 & 78 & 4 & 50 \\
\hline $\begin{array}{l}\text { Asie centrale et occidentale } \\
\text { (avec Turquie Transcaucasie } \\
\text { et Iran) }\end{array}$ & 310 & 6 & 84 & 10 & 250 \\
\hline $\begin{array}{l}\text { Sous-continent indien et Asie } \\
\text { du Sud-Est }\end{array}$ & 950 & 5 & 90 & 5 & 800 \\
\hline $\begin{array}{l}\text { Chine (avec Mongolie et } \\
\text { Corée du Nord) }\end{array}$ & 515 & 7 & 86 & 7 & 400 \\
\hline Japon et "dragons" & 140 & 18 & 53 & 29 & 50 \\
\hline Australasie et Océanie & 30 & 22 & 70 & 8 & 20 \\
\hline Monde entier & 3462 & 8 & 70 & 22 & 2200 \\
\hline
\end{tabular}

* sans compter l'évaporation des retenues

*** y compris l'évaporation des retenues

*** y compris des réutilisations et des productions d'eau non conventionnelles

Un tableau de ces utilisations agrégeant les pays par grands espaces économiques - comme on l'a fait pour les ressources - en atténuant de ce fait les incertitudes de nombreuses statistiques nationales, mais distinguant les principaux secteurs d'utilisation, a pu néanmoins être dressé (tabl. 6). Il fait ressortir les différences de structure des demandes suivant ces ensembles, ainsi que le poids largement prédominant de l'irrigation ; il propose aussi une estimation des consommations finales (volumes d'eau non restitués après usage : évapo-transpirés ou rejetés en mer).
Il est instructif par ailleurs de rapporter ces volumes annuels aux populations, comme on l'a fait pour les flux de ressource naturels : d'abord pour mesurer grossièrement la variété des demandes dans le monde et apprécier les incidences des deux autres facteurs, puis pour les comparer aux ressources, au plan des quantités. Malgré le fort nivellement opéré par un tel ratio globalisé par pays, surtout pour les plus peuplés, les demandes en eau par tête calculées varient dans une gamme très étendue : la moyenne mondiale actuelle, de l'ordre de $600 \mathrm{~m}^{3} / \mathrm{an}$ par 
Tableau 7. - Classes d'utilisation d'eau par habitant.

$\begin{array}{lc}\text { Pays } & \text { en } \mathrm{m}^{3} / \mathrm{an} \text { par habitant } \\ \text { surconsommateurs, à demande très forte } & >2000 \\ \text { gros consommateurs, à demande forte } & 1000 \text { à } 2000 \\ \text { modérément consommateurs, à demande moyenne } & 500 \text { à } 1000 \\ \text { peu consommateurs, à demande faible } & 100 \text { à } 500 \\ \text { sous-consommateurs, à demande très faible } & <100\end{array}$

tête, n'est qu'un point de repère théorique dans une gamme allant de moins de 50 à plus de $5000 \mathrm{~m}^{3} / \mathrm{an}$. On peut en déduire une échelle des utilisations d'eau en quantité, suivant laquelle les pays peuvent être classés indépendamment de leur population et les utilisations d'eau des pays peuvent être appréciées relativement comme fortes ou faibles par rapport à celle d'une classe médiane (tableaux 7 et 8 ).

Une géographie macroéconomique des utilisations d'eau peut s'esquisser sur cette base (fig. 4) et être comparée à celle des ressources.

Les demandes par habitant les plus fortes se trouvent dans les pays où l'irrigation pèse très largement dans les utilisations (Asie centrale ex-soviétique, Iraq, Pakistan, Madagascar, Iran, Egypte) aussi bien que dans des pays industrialisés très développés (Etats-Unis, Canada), les deux facteurs pouvant se combiner (Etats-Unis, Espagne, Argentine, Australie). Les principaux pays industrialisés où l'irrigation est négligeable, ou non dominante parmi les demandes, se rangent dans la classe des demandes modérées (RFA, France, Japon, Royaume Uni, Russie d'Europe) aux côtés de pays où le poids des populations réduit le ratio malgré l'importance des irrigations (Chine, Inde). Enfin, les demandes les plus faibles se trouvent soit dans des pays où elles sont plafonnées par les ressources - en zone aride, malgré la nécessité d'irrigation (Libye, Tunisie) ou dans des îles (Malte, Singapour) -, soit en pays peu développés en zone tropicale humide (Afrique centrale). Il va sans dire que, dans le cas de pays très étendus diversifiés en climat et en répartition de la population (Etats-Unis, Chine, Russie...), le calcul d'un index unique masque de fortes différences régionales internes.

Les structures des demandes dépendent largement d'autres facteurs que du niveau de développement socioéconomique, notamment du climat. De ce fait, on n'observe pas entre le volume des demandes en eau totales et un indicateur de développement tel que le P.N.B. de relation aussi nette que celle connue, par exemple, entre le P.N.B. et la consommation d'énergie, qui fait apparaître une élasticité positive. La comparaison tentée entre les quantités d'eau prélevées annuelles et les P.N.B. ${ }^{(4)}$ d'une cinquantaine de pays (années de la décennie 80), illustrée par la figure 5 , le met en évidence.

(4) en prenant cet indicateur pour ce qu'il vaut... et en sachant qu'il n'a sans doute pas la même signification pour tous les pays et qu'il prend inégalement en compte les activités utilisatrices d'eau non marchande.

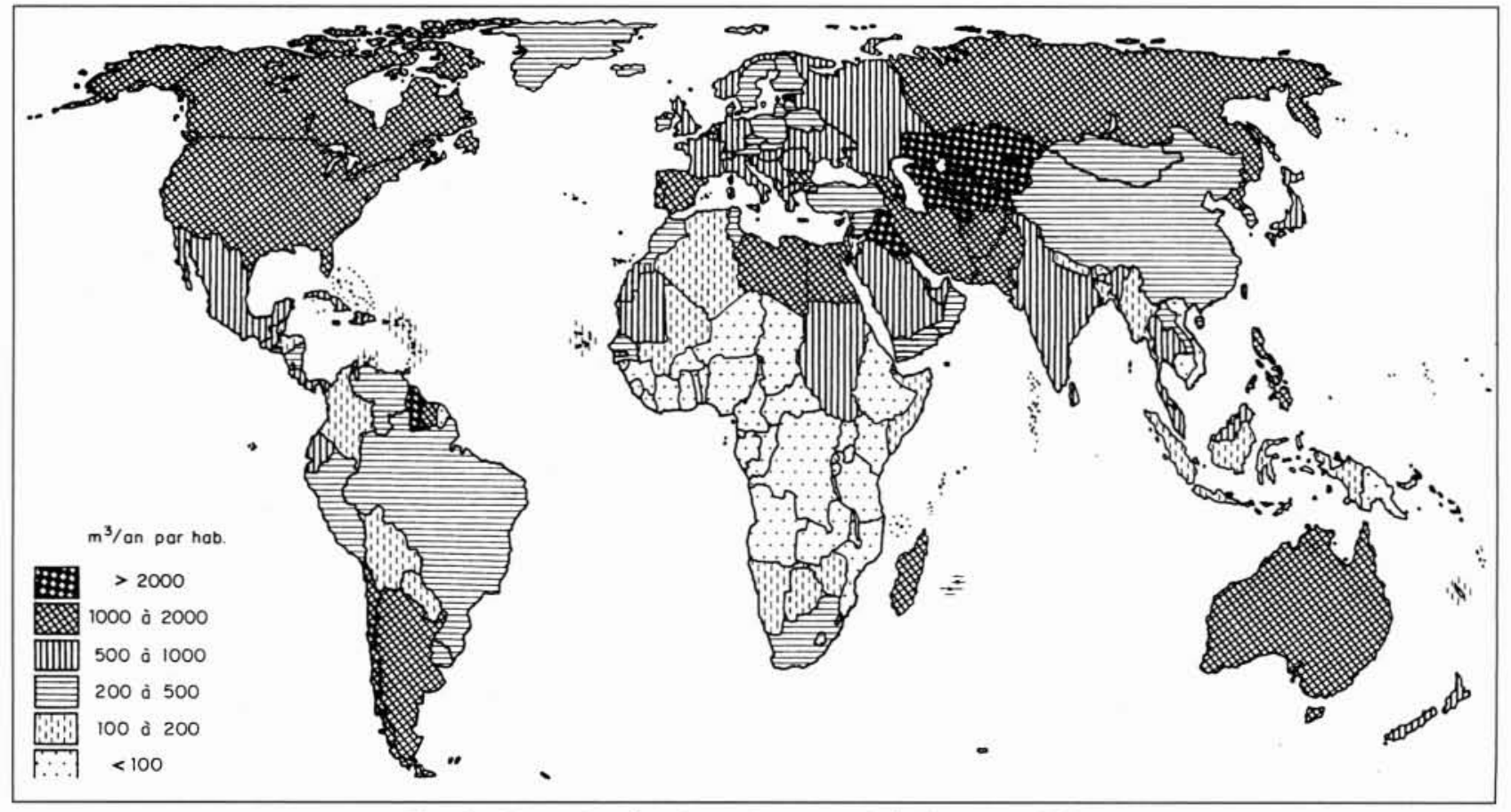

Fig. 4 - Pays classés suivant les quantités d'eau produites

(prélèvements, pour l'essentiel) par habitant, pour toutes les utilisations (1990 ou année proche). 
La dispersion des grandeurs des demandes à P.N.B. égal - ou inversement - paraît plutôt assez révélatrice du peu de relation entre les quantités d'eau produites pour satisfaire les demandes des différentes branches d'activité économique et les contributions de celles-ci à la formation du P.N.B. Il n'est que de comparer sous les deux angles l'agriculture irriguée, notamment, à diverses branches d'industrie à forte "valeur ajoutée". La figure 5 montre bien que, dans une colonne à P.N.B. sensiblement égal, les productions d'eau totales sont d'autant plus élevées que l'agriculture irriguée pèse plus dans la demande en eau.
Cette dispersion reflète donc bien la diversité des structures de demande en eau à un même niveau de développement économique. Par contre, la comparaison entre pays de structure similaire fait mieux ressortir la relation entre quantité d'eau utilisée et niveau de développement : par exemple, le groupe de pays à important secteur d'irrigation traditionnelle à demande en eau très prédominante (cf. Soudan, Egypte, Pakistan, Inde) ou celui des pays industrialisés où l'agriculture est peu ou non irriguée et ne contribue que pour une part minoritaire à la formation du P.N.B.

Tableau 8. - Pays classés suivant leur richesse et leurs "dépenses" en eau actuelles.

\begin{tabular}{|c|c|c|c|c|c|c|}
\hline \multirow{2}{*}{$\begin{array}{r}\text { Richesse en cau } \\
\text { (Ressource) } \\
\text { en m } \mathrm{m}^{3} / \mathrm{an} \\
\text { par hab. } \\
\text { (1995). } \\
\text { Utilisations } \\
\text { d'eau } \\
\text { (productions) } \\
\text { en } \mathrm{m}^{3} / \text { an par hab. }\end{array}$} & \multicolumn{2}{|c|}{$\begin{array}{l}\text { Seuil de } \\
\text { pénurie }\end{array}$} & $\begin{array}{l}\text { Seuil de } \\
\text { pauvreté } \\
\downarrow\end{array}$ & \multirow[b]{2}{*}{$\begin{array}{l}\text { Pays moyen- } \\
\text { nement dotés } \\
2000 \text { à } 10000\end{array}$} & \multirow[b]{2}{*}{$\begin{array}{c}\text { Pays riches } \\
10000 \text { à } 100000\end{array}$} & \multirow[b]{2}{*}{$\begin{array}{c}\text { Pays } \\
\text { très riches } \\
>100000\end{array}$} \\
\hline & $\begin{array}{l}\text { Pays très pauvres } \\
\quad<500\end{array}$ & $\begin{array}{l}\text { Pays pauvres } \\
500 \text { à } 1000\end{array}$ & $\begin{array}{l}\text { Pays restreints } \\
1000 \text { à } 2000\end{array}$ & & & \\
\hline $\begin{array}{l}\text { Très faible } \\
\quad<100\end{array}$ & $\begin{array}{c}\text { Malte } \\
\text { Singapour } \\
\text { Bahamas }\end{array}$ & Kenya & $\begin{array}{c}\text { Burkina Faso } \\
\text { Ethiopie }\end{array}$ & $\begin{array}{l}\text { Côte-d'Ivoire } \\
\text { Tanzanie } \\
\text { Nigéria } \\
\text { Ghana }\end{array}$ & $\begin{array}{c}\text { Zaïre } \\
\text { Congo } \\
\text { Cameroun } \\
\text { Tchad } \\
\text { Angola } \\
\text { Viet-Nam } \\
\text { Indonésie }\end{array}$ & $\begin{array}{l}\text { Gabon } \\
\text { Papua }\end{array}$ \\
\hline $\begin{array}{c}\text { Faible } \\
100 \text { à } 500\end{array}$ & $\begin{array}{c}\text { Algérie } \\
\text { Tunisie } \\
\text { Jordanie } \\
\text { Emirats } \\
\text { Arabes Unis } \\
\text { Qatar } \\
\text { Israël } \\
\text { Yémen } \\
\text { Gaza }\end{array}$ & Cap vert & $\begin{array}{c}\text { Afrique du Sud } \\
\text { Sénégal } \\
\text { Haüti } \\
\text { Pologne } \\
\text { Oman } \\
\text { Maroc } \\
\text { Liban } \\
\text { Tchéquie } \\
\text { Somalie } \\
\text { Zimbabwe }\end{array}$ & $\begin{array}{l}\text { Chine } \\
\text { Belarus } \\
\text { Ethiopie }\end{array}$ & $\begin{array}{c}\text { Suède } \\
\text { Autriche } \\
\text { Mali } \\
\text { Bangla Desh } \\
\text { Bolivie } \\
\text { Brésil } \\
\text { Colombie } \\
\text { Vénézuela }\end{array}$ & $\begin{array}{c}\text { Islande } \\
\text { Guyane (Fr.) }\end{array}$ \\
\hline $\begin{array}{c}\text { Modérée } \\
500 \text { à } 1000\end{array}$ & $\begin{array}{c}\text { Arabie Saoudite } \\
\text { Libye }\end{array}$ & 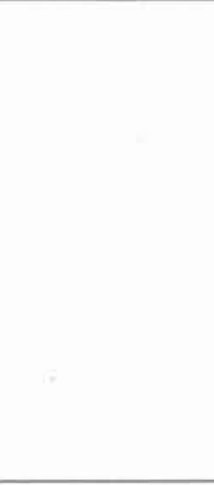 & $\begin{array}{l}\text { Belgique } \\
\text { Ukraine } \\
\text { Chypre }\end{array}$ & $\begin{array}{c}\text { France } \\
\text { RFA } \\
\text { Espagne } \\
\text { Italie } \\
\text { U.K. } \\
\text { Japon } \\
\text { Pays-Bas } \\
\text { Mexique } \\
\text { Pérou } \\
\text { Cuba } \\
\text { Syrie } \\
\text { Inde } \\
\text { Soudan } \\
\text { Suisse } \\
\text { Turquic } \\
\end{array}$ & $\begin{array}{c}\text { Russie } \\
\text { ex. Yougoslavie } \\
\text { Malaisie } \\
\text { Albanie } \\
\text { Nouv. Zélande }\end{array}$ & $\begin{array}{c}\text { Norvège } \\
\text { Alaska (USA) }\end{array}$ \\
\hline $\begin{array}{c}\text { Forte } \\
1000 \text { à } 2000\end{array}$ & & Egypte & Pakistan & $\begin{array}{c}\text { Bulgarie } \\
\text { Soudan } \\
\text { Iran } \\
\text { Afghanistan } \\
\text { Philippines } \\
\text { USA }\end{array}$ & $\begin{array}{l}\text { Canada } \\
\text { Chili } \\
\text { Argentine } \\
\text { Madagascar } \\
\text { Australie }\end{array}$ & \\
\hline $\begin{array}{l}\text { Très forte } \\
>2000\end{array}$ & & $\begin{array}{l}\text { Lower Colorado } \\
\text { (USA) }\end{array}$ & & $\begin{array}{c}\text { Azerbaidjan } \\
\text { Kazakhstan } \\
\text { Ouzbekistan } \\
\text { Iraq }\end{array}$ & $\begin{array}{l}\text { Turkmenistan } \\
\text { Up. Colorado } \\
\text { (USA) }\end{array}$ & $\begin{array}{c}\text { Sibérie } \\
\text { (Russie) } \\
\text { Suriname }\end{array}$ \\
\hline
\end{tabular}


Fig. 5 - Relation entre les demandes en eau totales et les P.N.B. en différents pays (années 1980-1990). Echelles logarithmiques. Pour chaque pays les dates de référence des deux variables sont cohérentes.

\section{III $\square$ UNE GÉOGRAPHIE HYDRO- ÉCONOMIQUE DIVERSIFIÉE ET CONTRASTÉE}

De la double diversité de répartition des ressources et des utilisations d'eau présentes, mêmes estimées sommairement par pays entier, résulte une grande variété de situations dans le monde. Le croisement des gammes respectives de "richesse en eau" et de "dépenses en eau" rapportées aux populations définies précédemment, permet de classer les pays suivant une typologie très différenciée : des pays riches en eau sont dépensiers ou économes (parfois par force, faute de moyens), des pays moyennement pourvus sont très inégalement utilisateurs, des pays pauvres utilisent tantôt beaucoup, tantôt peu d'eau (cf. tabl. 8).

\section{IV — L'AGGRAVATION À VENIR DES DISPARITÉS}

Même dans l'hypothèse où les ressources en eau naturelles resteraient stables en moyenne, les seules différences de croissance démographique auront pour premier effet de creuser les écarts entre les ressources par habitant de chaque pays, en diminuant celles-ci d'autant plus que les populations augmenteront, donc en transformant sensiblement la répartition de ces ressources unitaires. A l'horizon 2025, selon les prévisions démographiques médianes des Nations Unies, ces ressources pourraient être divisées par un coefficient variant de 1 à 3 suivant les pays ; elles seraient généralement divisées par 2 ou plus en Afrique. C'est souvent dans les pays où ces ressources sont à présent les plus faibles qu'elles risquent de diminuer le plus (pays de zone aride d'Afrique et du Moyen-Orient).

De plus l'enchérissement des coûts de mobilisation et de production de l'eau sera lui aussi très inégal : ces coûts augmenteront d'autant plus que les ressources en eau encore disponibles se raréfieront et qu'une partie appréciable des approvisionnements en eau devra être assurée par des ressources non conventionnelles. Là encore ce sont souvent des pays de zone aride - peu développés ou bénéficiaires de rente pétrolière - qui auront à supporter les coûts les plus croissants, ce qui tendra à modérer les demandes en eau réelles mais parfois aussi à handicaper le développement.

Les demandes en eau totales de chaque pays devraient, elles aussi, évoluer différemment, non seulement en fonction des croissances des populations, mais aussi du développement économique et parfois des changements

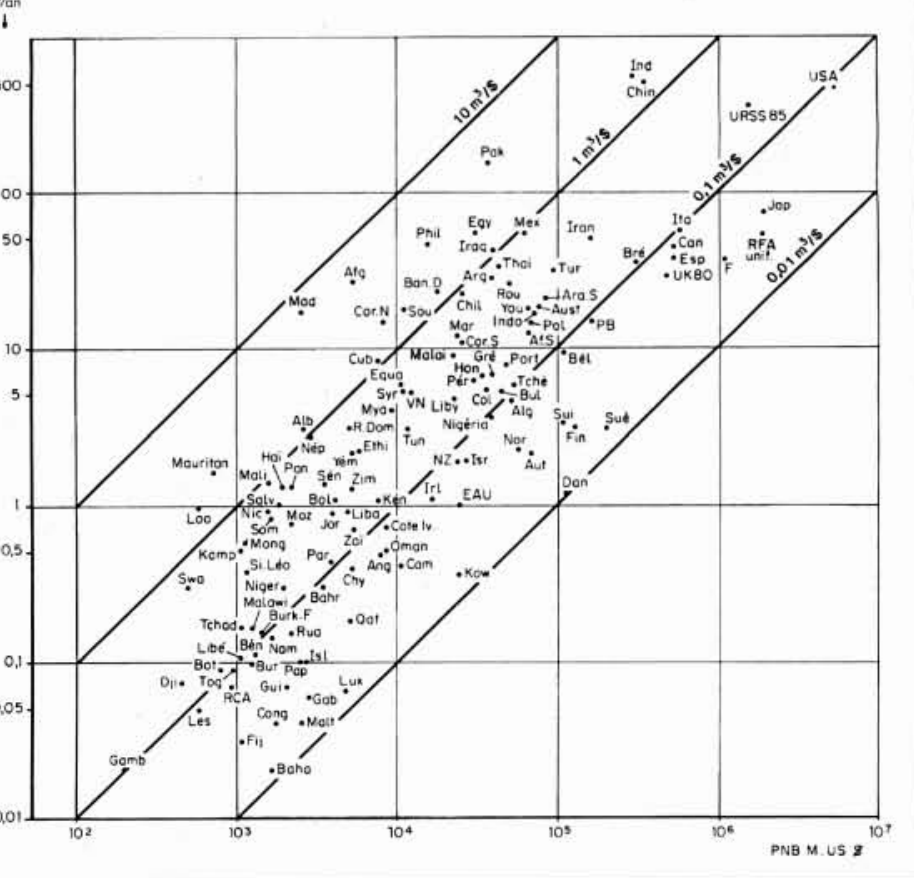

techniques. Elles pourraient croître assez inégalement, suivant l'évolution des besoins et des moyens de les satisfaire et aussi les plafonnements imposés par les ressources, ou parfois décroître sous l'effet de changement de leur composition relative. De ce fait, les demandes par habitant devraient évoluer très diversement suivant les pays : tantôt elles pourraient encore s'accroître, tantôt elles devraient inéluctablement décroître, ce qui aggravera leur disparité dans le monde. En Egypte, par exemple, les demandes en eau par habitant devraient diminuer de 20 à $40 \%$ entre 1995 et 2025 , suivant l'exercice de prospective du Plan Bleu (1996).

Enfin, les conséquences d'éventuels changements de climat à plus ou moins long terme, ne peuvent être éludées, malgré les incertitudes encore fortes qui affectent les modélisations globales du climat et les divergences de pronostic des experts, sur les ampleurs et les échéances des incidences sur le cycle de l'eau. Il serait en tout cas imprudent de tabler sur une stationnarité du climat et des régimes moyens des eaux. Des écarts plus accentués entre les climats humides et arides sont à présager, ainsi que des irrégularités accrues des apports entre les saisons et les années. En toute hypothèse, une aridification possible dans certaines parties du monde (domaine méditerranéen notamment) aurait pour double effet de raréfier les ressources et de dégrader leur maîtrisabilité, tout en amplifiant les besoins, ce qui risque d'accentuer encore les inégalités de situation entre les pays.

Les différences de tension présentes entre les demandes en eau et les ressources, suivant les pays, pourraient ainsi s'amplifier fortement à l'avenir. Il en découlera des degrés variés de pression sur les eaux naturelles - par les prélèvements et les rejets -, donc d'artificialisation du régime des eaux, dont la majeure partie sera impliquée dans la 
sphère économique, dans des pays en nombre croissant. De même, le contraste sera plus tranché entre les économies de l'eau traditionnelles, dominantes encore dans une grande partie du monde, et les nouvelles économies de l'eau, amorcées aujourd'hui mais qui seront plus développées à l'avenir dans certains pays, basées davantage sur les ressources non conventionnelles et une gestion de l'eau devenue un produit industriel.

Les charges économiques d'aménagement des eaux, d'approvisionnement et de conservation des ressources sont encore, à présent, fort inégales, tant dans l'absolu que par rapport au P.N.B. de chaque pays. Elles vont croître aussi inégalement, souvent plus vite que les P.N.B., notamment du fait de l'élévation des coûts en fonction des taux d'exploitation des ressources et de la part prise par les productions d'eau non conventionnelles, en amplifiant par conséquent les différences de leur poids relatif dans les économies nationales. Les pays où les charges sont les plus fortes et risquent de croître le plus sont souvent les plus pauvres. Cela peut handicaper le développement des pays les plus dépourvus de ressource et à faible revenu, et faire obstacle, en particulier, aux politiques d'autosuffisance alimentaire souvent voulues. Cela peut fausser les compétitions économiques, notamment dans le marché des produits agricoles.

La pauvreté sera le principal obstacle à la couverture des besoins en eau - pour l'alimentation en eau potable, notamment des grandes agglomérations, et pour l'irrigation - dans une partie du monde en extension, y compris dans des pays à ressources en eau abondantes.

Les situations de pénurie favorisent les conflits d'usage entre les secteurs d'utilisation, en particulier entre les utilisations d'eau marchande et d'eau non marchande (ou très subventionnée) : en pratique entre l'alimentation en eau urbaine et l'irrigation. La diminution des parts de ressource allouée à l'agriculture irriguée est une tendance générale, dans beaucoup de pays ; elle impose à cette agriculture de fortes transformations des procédés d'irrigation et des objectifs de production, pour éviter un dépérissement. Des conflits vont aussi s'aggraver entre l'ensemble des utilisations socio-économiques et la préservation de milieux naturels, en particulier des zones humides.

Enfin, les situations de pénurie vont exacerber les conflits de répartition des ressources en eau communes à plusieurs pays, voire à plusieurs régions dans un même pays, tout particulièrement dans les bassins fluviaux ou les grands aquifères transfrontaliers en zones aride et semi-aride.

\section{BIBLIOGRAPHIE ESSENTIELLE}

[1] Andreassian V. et Margat J. (1996) - Prospective des besoins en eau mondiaux à thorizon 2025. (Coll. intern. "Quel environnement au XXIe siècle ? Environement. maîtrise du long terme et démocratie". Fontevraud, septembre, 31 p.).

[2] Engelman R. and Leroy P. (1993) - Sustaining Water. Population and the Future of Renewable Water Supplies (Population and Environment Program/Population Action International, Washington, 56 p.).

[3] Falkenmark M. (1986) - Macro-Scale Water Supply/Demand Comparison on the Global Scene (Beitrage Zur Hydrologie - Sonder. 6, pp. 15-40 - Stockholm).
[4] FAO (1995) - Water Resources of African Countries: A Review. (FAO, 35 p., Rome).

[5] FAO (1997) - Water Resources of the Near East Region: A Review. (FAO, 38 p., Rome).

[6] Gleick P.H. ed. (1993) - Water in Crisis - A guide tot the World Fresh Water Resources (Oxford University Press. New-York, Oxford. 449 p.).

[7] Juhasz F. (1989) - Y a-t-il une crise de l'eau ? (L'observateur de l'OCDE, $\mathrm{n}^{\circ}$ 160. Paris, octobre-novembre, pp. 4-9).

[8] L'Vovich M.I. (1977) - World water resources, present and future. (Ambio, vol. $6, \mathrm{n}^{\circ}$ I. p. 13-21, Stockholm).

[9] Margat J. (1987) - Les trois stades de l'économie de l'eau. (A.I.S.H., Proceed. Symp. "Water for the Future: Hydrology in perspective", Rome, Av. Publ. AISH n 164 . pp. 47-51).

[10] Margat J. (1994) - L'économie de l'eau dans le monde - Cons. écon. et soc., Acad, Sc/CADAS, FR., C.R. Journée sur "les aménagements hydrologiques", Paris, 26.05.1994, pp. 22-42, publ. Fév. 1995.

[11] Margat J. (1994) - Les utilisations d'eau dans le monde : état présent et essai de prospective. (Contribution au projet M-13 du PHI-IV/UNESCO, Paris. 87 p.).

[12] Margat J. (1995) - L'économie de l'eau dans le monde : ressources, besoins, problèmes. A chacun ses problèmes d'eau. (in: "Le grand livre de l'eau" 2nd éd. pp. 227-288. Ed. La Manufacture/Cité des Sciences et de I'Industrie, Paris.

[13] Margat J. (1996) - L'alimentation en eau de lhumanité. Situation et tendances présentes. Prospective. (Colloque "Leau et la vie des hommes au XXIè siècle", Mouvement Universel de la Responsabilité Scientifique/M.U.R.S.. Mars, Paris, 33 p.).

[14] Margat J. (1966) - Les ressources en eau. Conception, évaluation, cartographie, comptabilité (Ed. FAO/BRGM, Coll. Manuels et méthodes n²8, Orléans. 146 p.).

[15] Meybeck M. et al. (1989) - Global Freshwater Quality: a First Assessment (OMS-PNUE - Blackwell Reference, Oxford)

[16] Obasi G.O.P/O.M.M. (1997) - Changements climatiques et Gestion des ressources en eau douce. (Forum Mondial de l'Eau, Marrakech, 22 mars. Organisation météorologique mondiale, 16 p.).

[17] Plan Bleu/Benblidia M., Margat J., Vallée D. (1996) - L'eau en région méditerranéenne - Water in the Mediterranean Region. (Conférence Euroméditerranéenne sur la gestion de l'eau. Marseille, novembre 1996, rédit. Plan bleu. 91 p., 1997).

[18] Postel S. (1994) - Water: Rethinking Management in an Age of Scarcity. (Worldwatch Paper 62, New-York)

[19] Postel S. (1992) - Last Oasis, Facing water scarcity - New-York, London, Worldwatch Institute, Norton and Co., 240 p.

[20] Serageldin 1. (1995) - Towards Sustainable Management of Water Resources (The World Bank, Direction in Development, Washington, 33 p.).

[21] Shiklomanov 1.A. (1990) - The World Water Resources: How much do we really know about them ? (UNESCO. IHD/IHP, 25-year Commem. Sympos.. Paris 15-17 mars. Publ. 1991).

[22] Shiklomanov I.A. (1996) - Assessment of Water Resources and Water Availability in the World. (State hydrological Institute of Russia/UNESCO, February, 127 p.).

[23] Collectif (1992) - International Conference on Water and the Environment: development issues for the 21 st century (UN et al., Dublin, 26-31 janv. Keynote Papers, Dublin Statement and Report).

[24] Collectif (1997) - L'eau, patrimoine de l'humanité (contrib. au ler Forum mondial de l'eau, Marrakech, mars, Conseil mondial de l'eau).

[25] Anonyme/World Resources Institute (1996) - World Resources 1996-1997. Part II, 8 Freshwater and Table/13.1 (World Resources Institute/Intern. Inst. for Environment and Development, Washington).

[26] Anonyme/OMM-UNESCO (1997) - Y aura-t-il assez d'eau sur Terre ? (OMM-UNESCO, OMM n ${ }^{\circ} 857,22$ p.).

[27] Anonyme/UN (1997) - The Freshwater Resources of the World: A comprehensive Assessment - Challenge and Actions (UN Report, New-York).

[28] Anonyme/ONU et al. (1997) - Inventaire exhaustif des ressources mondiales en eau douce (OMM, Genève).

[29] Anonyme/OCDE (1997) - Données OCDE sur l'environnement - Compendium 1997, 3.1 A. B, C. Eaux intérieures (OCDE, pp. 66-75. Paris) 